\title{
Strengthening Patient-Centered Care in the VHA: A Relational Model of Change
}

\author{
Jody Hoffer Gittell, MA, PhD and Lauren Hajjar, MA, MPA, PhD
}

J Gen Intern Med 34(Suppl 1):S7-S10

DOI: $10.1007 / \mathrm{s} 11606-019-04996-7$

(c) Society of General Internal Medicine 2019

W idespread efforts have been dedicated to patientcentered care throughout the USA, in private healthcare systems as well as public healthcare systems like the Veterans Health Administration (VHA). ${ }^{1,2}$ Despite its intended promise, its scientific credibility, and its policy relevance, the successful implementation of patient-centered care has been uneven. ${ }^{3}$ In this article, we shed light on the uneven implementation of patient-centered care in the VHA and elsewhere and describe how the relational model of organizational change can help.

\section{RELATIONAL COORDINATION AND THE ROLE OF HUMAN RESOURCE PRACTICES}

Relational coordination theory proposes that coordination is most effectively carried out through frequent, timely, accurate, problem-solving communication among key stakeholders, including clients, supported by relationships of shared goals, shared knowledge, and mutual respect. Originally developed in the airline industry, the theory has been tested in the banking, software, construction, manufacturing, and education sectors, and in a wide array of healthcare settings. ${ }^{4}$ Overall, the research suggests that high-quality communication and relationships, supported by well-designed structures, are at the heart of successful patient-centered care. ${ }^{5}$

Some of the structures needed to support patient-centered care are already being implemented with a significant investment of resources by the VHA, including (1) boundary spanner roles like case managers and care coordinators, (2) shared information systems, (3) shared meetings, and (4) shared protocols. But the research suggests that more is needed. Coordinating patient-centered care also requires innovative HR practices such as (1) job descriptions that highlight the coordination responsibilities associated with each job, (2) systematically selecting employees for teamwork, (3) systematically training employees for teamwork, (4) systems of shared accountability and shared rewards, and (5) conflict

Published online May 16, 2019 resolution to address differences that are bound to emerge as participants strive to coordinate their work in new ways.

These kinds of structures or high performance work systems have been adopted by industry leaders in many sectors including autos, banking, apparel, telecommunications, airlines, and more recently healthcare. ${ }^{6-9}$ Together, these structures work to support the coordination of patient care across functional, departmental, and organizational boundaries. When these structures are in place, patient-centered care can be carried out reliably and at scale. Without support from these structures, patient-centered care depends upon the individual efforts of highly mission-driven employees. These individual efforts can be exhausting in the absence of the necessary structural supports. Dedicated employees who engage in these individual efforts may feel a bit like Sisyphus, rolling a rock up a mountain each day only to see it roll back down. ${ }^{1}$

In sum, patient-centered care is challenging to implement when it runs up against the silos that are unintentionally reinforced by inherited HR practices. Even when appropriate coordinating mechanisms are designed and implemented - case management roles, shared information systems, shared meetings, and shared protocols that cut across the relevant boundaries to coordinate patient-centered care - inherited HR practices invisibly create resistance in the opposite direction.

\section{GETTING FROM HERE TO THERE}

To get from here to there, we suggest partnerships between the Department of Human Resources and operational leaders using the relational model of organizational change ${ }^{9,}{ }^{10}$ (see Fig. 1). Informed by research and close observations of change efforts, this model includes a six-stage process of participatory, multistakeholder change, summarized as follows:

Stage 1: Explore Context. Identify patient care coordination challenges and desired outcomes. Key stakeholders involved in these coordination challenges, including Veterans and family members, are introduced to the principles of relational coordination.

"According to the Greek myth, Sisyphus is condemned to roll a rock up to the top of a mountain, only to have the rock roll back down to the bottom every time he reaches the top. The gods were wise, [philosopher Albert] Camus suggests, in perceiving that an eternity of futile labor is a hideous punishment," Summary, The Myth of Sisyphus. 


\section{Middle \& Top Leadership}

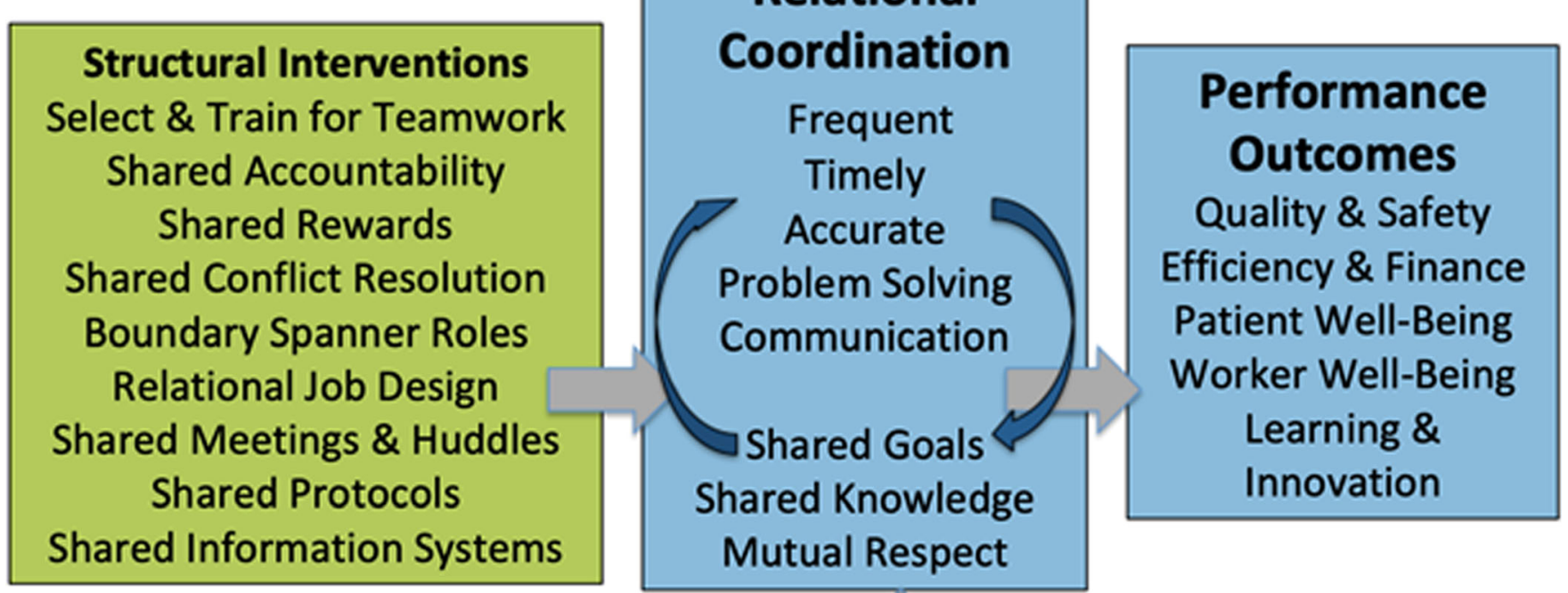

Frontline Leaders, Co-Workers \& Patients

\section{Relational Interventions Create Safe Space Relational Assessment Humble Inquiry/Coaching}

Work Process Interventions Assess Current State Identify Desired State Experiment to Close the Gap

Figure 1 Relational model of organizational change.

Stage 2: Form Change Teams. Multi-stakeholder change teams are formed to further identify coordination challenges through relational mapping. Facilitators create a safe space for stakeholders with different knowledge, status and power to disagree respectfully and come to agreement.

Stage 3: Measure RC. Change teams assess coordination challenges systematically using a validated measurement tool called the Relational Coordination Survey. $^{11}$

Stage 4: Reflect on Findings. Change teams receive visual feedback of RC data revealing strengths and opportunities. These visual representations help change teams to develop a common understanding of the current state, and provide a starting point for new conversations, collective sense-making, reflection, and change ${ }^{22}$ (see Fig. 2).

Stage 5: Design Interventions. Change teams design and implement interventions to address the opportunities they have identified in the data. Relational interventions such as conversations of interdependence and humble inquiry help to shift the culture from fragmented silos to connected networks based on shared goals, shared knowledge, mutual respect, and communication that is sufficiently frequently, timely, accurate, and focused on problem-solving rather than blaming. Structural interventions include the redesign of HR practices, such as updated job descriptions that articulate coordination responsibilities associated with each job, hiring and training criteria that address these coordination responsibilities, shared accountability and shared rewards for outcomes, and new processes for resolving conflicts, in addition to strengthening the boundary spanner roles, shared information systems, shared meetings, and shared protocols that have already been developed to support patient-centered care. Work process interventions provide tools to guide the improvement process and redesign workflows as needed (see Fig. 3).

Stage 6: Assess and Adjust. Change teams conduct periodic assessments to evaluate progress and to recommend additional interventions to further strengthen patient-centered care where needed.

\section{IMPLICATIONS FOR THE VHA}

Coordination challenges involved in delivering patientcentered care have multiplied for the VHA due to the increasingly complex medical, mental health, and social conditions of Veterans. Coordination challenges have also multiplied due to 


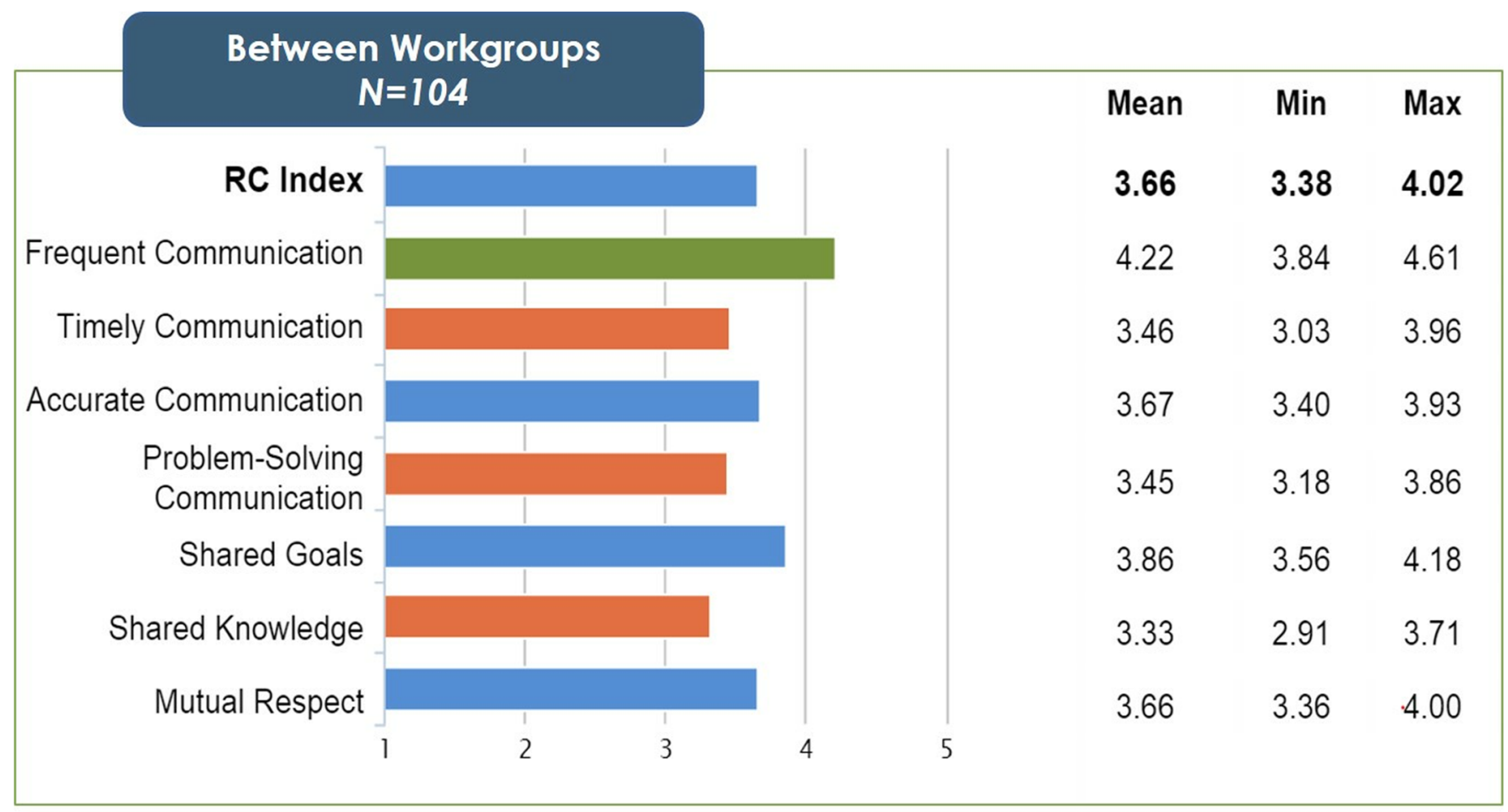

(C) 2016 Relational Coordination Analytics, Inc. All Rights Reserved

Figure 2 Relational Coordination Survey data.

the federally mandated policy that allows Veterans to receive care both within and outside the VHA system, with the expectation that their care will continue to be coordinated by the VHA. The relational model of organizational change described above is particularly relevant for meeting these challenges because, while it is about building relationships to coordinate care, it is also about redesigning structures to systematically and reliably support these relationships. Some

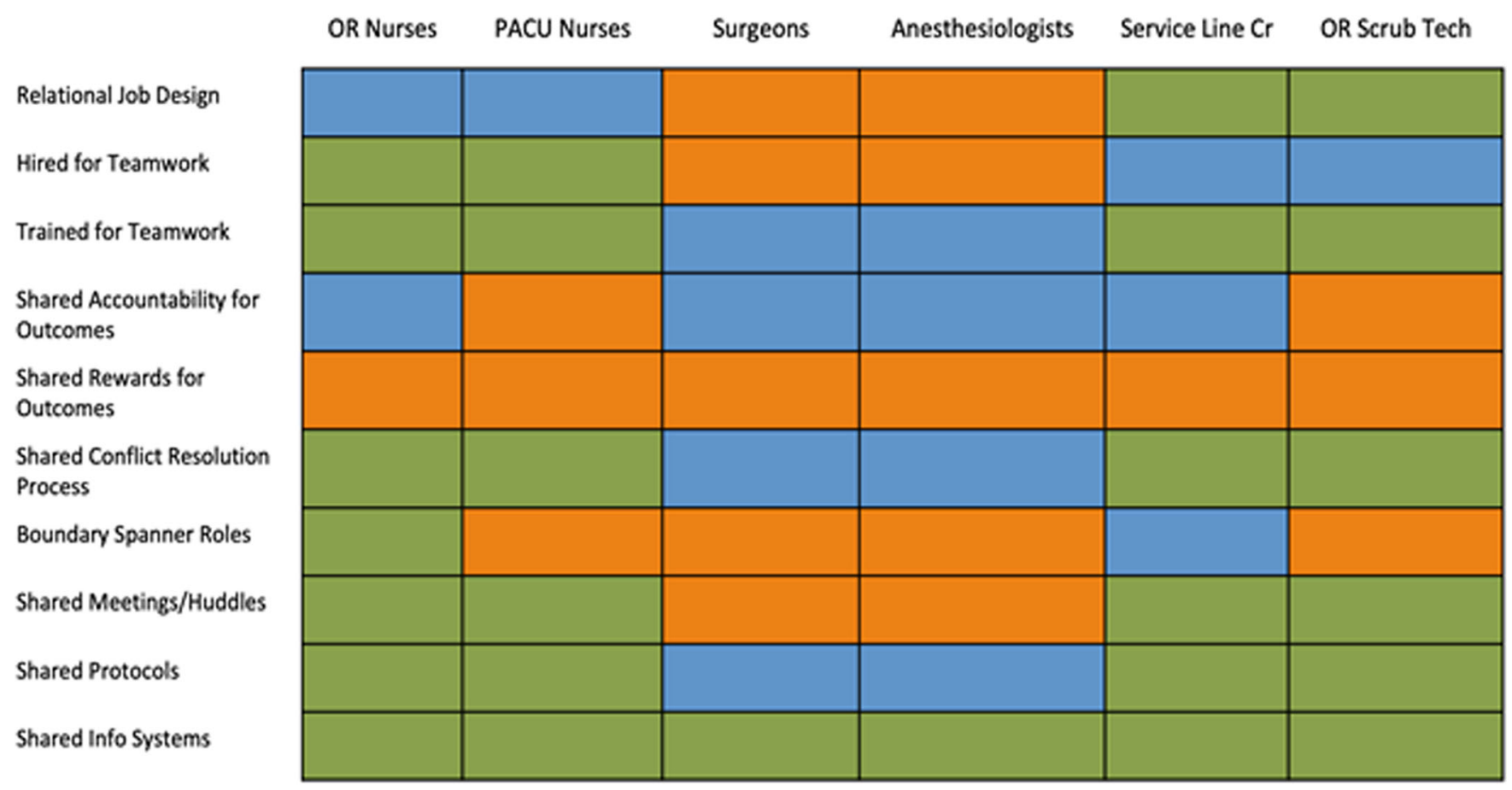


structures can be redesigned locally. ${ }^{10}$ But these local redesigns will be hard to sustain until they become the basis for a system-wide redesign of $\mathrm{HR}$ practices.

For organizations like the VHA that are characterized by both centralization and decentralization, we propose a change process described above that is both bottom up and top down, led by frontline leaders and backed by central leaders. This process could start with a handful of sites, with facilitators provided by the Department of Human Resources, protected time to form multi-stakeholder change teams and to engage in the six stages of change, and a virtual space to share their learning with each other. This process would be evaluated to determine whether HR practices were indeed changed, whether relational coordination was strengthened, and whether outcomes such as employee engagement, Veteran engagement, quality and safety were improved. Lessons learned will suggest a path for spreading these changes more broadly across the VHA, in partnership with the Department of Human Resources, to better support the mission of patient-centered care.

Corresponding Author: Jody Hoffer Gittell, MA, PhD; Brandeis University, 415 South St. MS 035, Waltham, MA 02453, USA (e-mail: jgittell@brandeis.edu).

\section{REFERENCES}

1. Weiner SJ, Schwartz A, Sharma G, et al. Patient-centered decision making and health care outcomes: An observational study. Ann Intern Med. 2013;158:573-9.
2. Bokhour BG, Fix GM, Mueller NM, Barker AM, Lavela SL, Hill JN, ... \& Lukas CV. How can healthcare organizations implement patient-centered care? Examining a large-scale cultural transformation. BMC Health Serv Res. 2018;18(1):168.

3. Rathert C, Wyrwich MD, Boren SA. Patient-centered care and outcomes: a systematic review of the literature. Med Care Res Rev. 2013;70:351-79.

4. Gittell JH, Fairfield K, Bierbaum B, Jackson R, Kelly M, Laskin R, Lipson S, Siliski J, Thornhill T, Zuckerman J. Impact of relational coordination on quality of care, post-operative pain and functioning, and length of stay: A nine hospital study of surgical patients. Med Care. 2000;38(8):807-819.

5. Gittell JH. High performance healthcare. New York, NY: McGraw Hill;2009.

6. MacDuffie JP. Human resource bundles and manufacturing performance: Organizational logic and flexible production systems in the world auto industry. ILR Rev. 1995;48(2):197-221.

7. Batt $\mathbf{R}$. Work design, technology and performance in customer service and sales. ILR Rev. 1999;52:539-564.

8. Gittell JH. The Southwest Airlines way: Using the power of relationships to achieve high performance. New York, NY: McGraw-Hill;2003.

9. Gittell $\mathbf{J H}$. Transforming relationships for high performance: The power of relational coordination. Palo Alto, CA: Stanford University Press;2016.

10. Leonard C, Lawrence E, McCreight M, Lippmann B, Kelley L, Mayberry A, ... Jones J. Implementation and dissemination of a transition of care program for rural veterans: A controlled before and after study. Implement Sci. 2017;12(1):123.

11. Valentine M, Nembhard I, \& Edmondson A. Measuring teamwork in health care settings: A review of survey instruments. Med Care. 2015;53(4):e16-e30.

Publisher's Note Springer Nature remains neutral with regard to jurisdictional claims in published maps and institutional affiliations. 